\title{
Resonant Tunneling in Nanocolumns Improved by Quantum Collimation
}

\author{
Jakob Wensorra, ${ }^{\dagger}$ Klaus Michael Indlekofer, ${ }^{*, \dagger}$ Mihail Ion Lepsa, ${ }^{\dagger}$ \\ Arno Förster, ${ }^{\mathrm{t} \neq}$ and Hans Luth ${ }^{\dagger}$
}

Institute of Thin Films and Interfaces (ISG-1) and Center of Nanoelectronic Systems
for Information Technology (CNI), Research Centre Jülich, D-52425 Jülich, Germany,
and Aachen University of Applied Sciences, Section Jülich, D-52428 Jülich, Germany

Received September 6, 2005; Revised Manuscript Received October 10, 2005

\begin{abstract}
We report on a quantum collimation effect based on surface depletion regions in AIAs/GaAs nanocolumns with an embedded resonant tunneling structure. The considered MBE-grown nanodevices have been fabricated by means of a top-down approach that employs a reproducible lithographic definition of the vertical nanocolumns. By analyzing the scaling properties of these nanodevices, we discuss how a collimation effect due to a saddle point in the confining potential can explain an improved device performance of the ultimately scaled structures at room temperature.
\end{abstract}

Quasi-one-dimensional nanostructures such as carbon nanotubes and semiconductor nanocolumns (whiskers) have attracted ample interest as candidates for novel nanodevice concepts. In fact, scaling of transistor designs down to the decananometer regime requires ultrathin or even $1 \mathrm{D}$ nanodevice concepts in order to preserve electrostatic integrity. ${ }^{1}$ In this sense, nanocolumns represent a perfect device system. They offer not only an ultimately scaled nanodevice structure in a beyond-CMOS scenario but also allow for the investigation of fundamental questions of electronic transport in quasione-dimensional quantum systems. By means of built-in heterostructures, one can easily implement tunnel barriers and quantum well structures within such nanocolumns. Although the fabrication of nanocolumns by self-organizing growth processes ${ }^{2-7}$ is certainly a very attractive feature, there are great challenges concerning the reproducible realization of identical devices for large circuits and wiring on a nanometer scale. It is therefore important to study also the top-down approach, which is based on a lithographic definition of vertical nanostructures. ${ }^{8-17}$ Hence, such an approach is presented in this paper, focusing on the scaling properties of nanostructured AlAs/GaAs resonant tunneling devices. We show how lateral surface depletion regions in such structures can result in a quantum collimation effect that gives rise to an unconventional but advantageous scaling behavior. In this context, space charge layers offer completely new design parameters for transport properties and hence for the performance of devices based on nanocolumns.

\footnotetext{
* Corresponding author. E-mail: m.indlekofer@fz-juelich.de.

†nstitute of Thin Films and Interfaces (ISG-1) and Center of Nanoelectronic Systems for Information Technology (CNI).

$\doteqdot$ Aachen University of Applied Sciences.
}

Resonant tunneling diodes (RTD), as they are studied in this paper, are essentially Fabry-Perot interferometers (FPI) for electron waves. ${ }^{18}$ The semitransparent mirrors of the FPI are represented by two heterostructure barriers in the conduction band of the semiconductor. For the present experiments, vertical GaAs nanocolumns with two embedded AlAs barriers (thickness $1.7 \mathrm{~nm}$ ) separated by a 5-nm layer of GaAs (quantum well) were processed by e-beam lithography from an MBE-grown layer stack (see Figure 1). The grown layer structure is shown in Figure 1c. Starting from an n-type GaAs substrate, 500-nm highly n-doped GaAs $\left(n^{+}=5 \times 10^{18} \mathrm{~cm}^{-3}\right)$ is grown with two subsequent $10-\mathrm{nm}$ layers of lower n-type doping. GaAs spacers clad the AlAs/ GaAs double-barrier structure, resulting in an undoped region with a total thickness of $18.4 \mathrm{~nm}$. The next layers consist of n-doped GaAs with increasing doping concentrations up to $n^{+}=5 \times 10^{18} \mathrm{~cm}^{-3}$. In this way, a symmetrical layer structure with respect to the center of the RTD quantum well is obtained. Finally, the epitaxial growth ends with a $10-\mathrm{nm}$ highly doped $n^{++}=2 \times 10^{19}$ layer and a thin $(2.8 \mathrm{~nm})$ undoped low-temperature grown (LTG) GaAs layer. This layer combination, $n^{2+}-\mathrm{GaAs} / \mathrm{LTG}-\mathrm{GaAs}$, together with a $\mathrm{Ti} / \mathrm{Au}$ metallization (deposited immediately after the epitaxial growth) provides an excellent nonalloyed shallow ohmic contact for nanodevices $\left(\rho_{\mathrm{C}}=(1-2) \times 10^{-5} \Omega \mathrm{cm}^{2}\right.$ in the present case). ${ }^{19,20}$ It is important to mention that all of the processed nanocolumn devices stem from the same wafer and hence possess an identical epitaxial layer stack. In turn, the nanocolumns with diameters of $50 \mathrm{~nm}$ to $1 \mu \mathrm{m}$ are defined by e-beam lithography using a high-resolution inorganic negative e-beam resist (Hydrogen Silsesquioxan, 

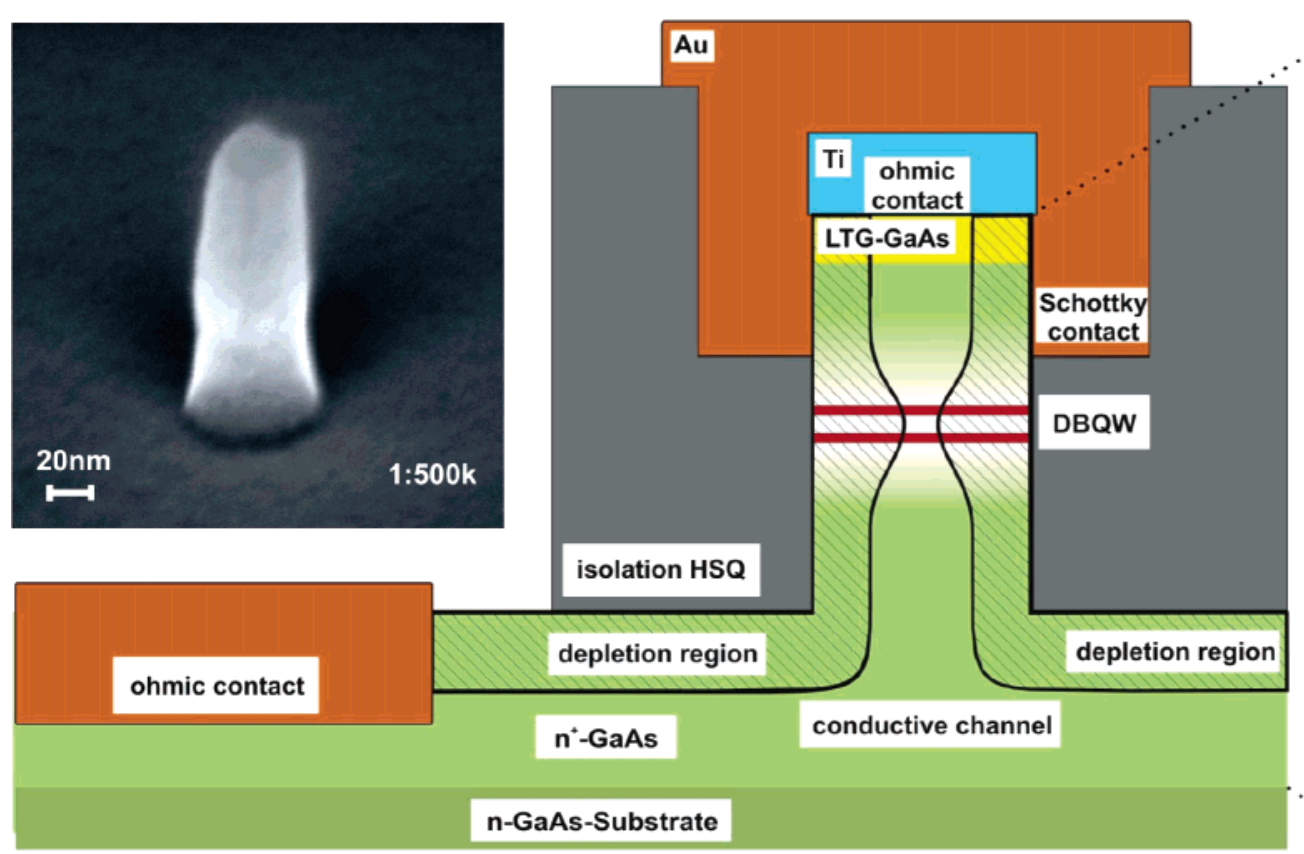

LTG-GaAs 2,8nm undoped

$10 \mathrm{~nm} \mathrm{n}^{+4}=2 \times 10^{19} \mathrm{~cm}$

$52 \mathrm{~nm} \mathrm{n}^{+}=5 \times 10^{18} \mathrm{~cm}$

GaAs

$10 \mathrm{~nm} \mathrm{n}=1 \times 10^{17} \mathrm{~cm}^{-3}$

$10 \mathrm{~nm} \mathrm{n}=1 \times 10^{16} \mathrm{~cm}^{-3}$

$5 \mathrm{~nm}$ undoped

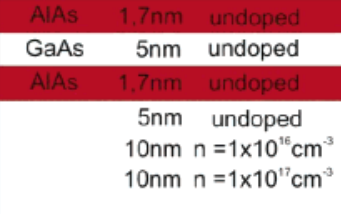

GaAs

$500 \mathrm{~nm} \mathrm{n}^{+}=5 \times 10^{18} \mathrm{~cm}$

Figure 1. (a) SEM micrograph of a 50-nm GaAs nanocolumn with ohmic contact and HSQ-mask on top. (b) Schematic cross-sectional view of the vertical device structure with an embedded double-barrier quantum well (DBQW) structure of an RTD. (c) Heterostructure layout with double-barrier (red) and contact regions (yellow).

$\mathrm{HSQ}^{21,22}$ ) and subsequent ion-beam and reactive-ion-beam (RIE) etching steps. In order to obtain a good aspect ratio, it is crucial to etch the semiconductor material in sequential $\mathrm{H}_{2} / \mathrm{CH}_{4}$ and $\mathrm{O}_{2}$ plasmas. Figure 1a shows a SEM micrograph of a 50-nm column with the HSQ-mask on top. Afterward, the samples are planarized with several HSQ layers that serve as an isolation for the top contact pad. By means of optical lithography, 5- $\mu \mathrm{m}$ windows are opened in the resist mask and a further RIE step is employed to expose the top contact of the nanocolumns. Here, accurate etch control is mandatory in order to achieve a well-defined vertical position of the final Au metallization layer, which is deposited subsequently. Figure 1b shows a schematic cross-section of the complete device structure with top and bottom ohmic contacts. Note that the Au metallization of the top contact exhibits a partial overlap (typically $60 \mathrm{~nm}$ ) with the upper section of the column. This causes the formation of a Schottky side-contact in the upper GaAs region (see Figure 1b), always having the same potential as the ohmic part of the top-contact. Furthermore, the spatial extension of the resulting (space charge) depletion regions at the surface of the nanocolumn and the substrate is sketched qualitatively in Figure $1 \mathrm{~b}$ as well. Within the undoped double-barrier region, the conductive vertical channel of the nanocolumn becomes narrower due to the extended depletion zone. A detailed analysis of the consequences of this additional confinement effect will be given below.

DC measurements at room temperature have been carried out on the processed nanodevices. The obtained currentvoltage $(I-V)$ characteristics for the smallest devices (50$110 \mathrm{~nm}$ in diameter) are shown in Figure 2. As a general feature, the measured $I-V$ curves exhibit a current peak and a region of negative differential resistance (NDR), which are typical for RTDs. This behavior results from a peaked transmission coefficient of the double-barrier structure (analogous to the Fabry-Perot interferometer) ${ }^{18}$ in conjunction with the injected electron distribution from the emitter. The peak-to-valley current ratio (PVR) serves as a figure of merit for RTDs.

As can be seen in Figure 2a, the peak positions in the $I-V$ characteristics shift to higher voltages with decreasing column diameters. This observation can be attributed to the partial formation of a depletion barrier for the smaller structures (see below), which is overcome by a higher bias voltage. Unexpectedly, however, the peak width of the 50nm column $\Delta V_{\mathrm{p}} \approx 160 \mathrm{mV}$ (counted at the half-height between the peak and the valley current) is significantly reduced compared to $\Delta V_{\mathrm{p}} \approx 300-450 \mathrm{mV}$ for the larger diodes (with diameters of $110 \mathrm{~nm}$ down to $80 \mathrm{~nm}$, see Figure 2). Apart from this, the PVR reaches an unusually high value of $\sim 2$ for the smallest nanocolumn (Figure $2 b$ ). The latter effect is even more evident if one plots the measured PVR values as a function of the column diameter (Figure 3). Note that the plotted data points correspond to ensemble averages over multiple devices of the same nominal size with an identical layer stack. Typically, with decreasing lateral dimension the PVR becomes smaller due to an enhanced surface scattering in comparison to bulk scattering, which results in a higher relative valley current, as observed for devices down to $70 \mathrm{~nm}$. Here, the unexpected interesting behavior is the sudden increase of the PVR for the 50-nm diodes.

To understand the peculiarities of the 50-nm nanocolumns, we have simulated the 2D-potential map of the structure by means of a self-consistent semiclassical drift-diffusion solver, ${ }^{24}$ assuming a cylindrical symmetry of the vertical 


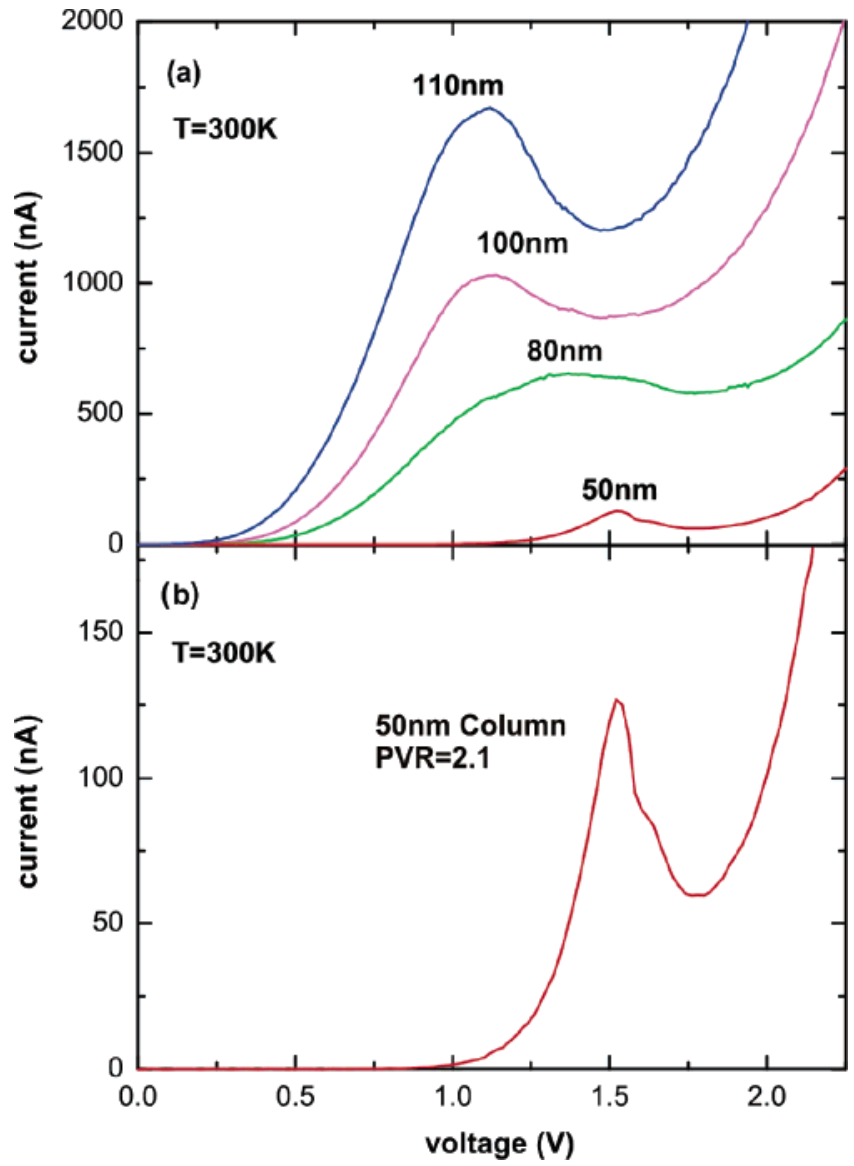

Figure 2. (a) Experimental $I-V$ characteristics for various lateral dimensions of the nanocolumns; here, the positive bias is applied to the top contact. (b) $I-V$ characteristics for the ultimately scaled 50-nm device (same as 50-nm curve in a).

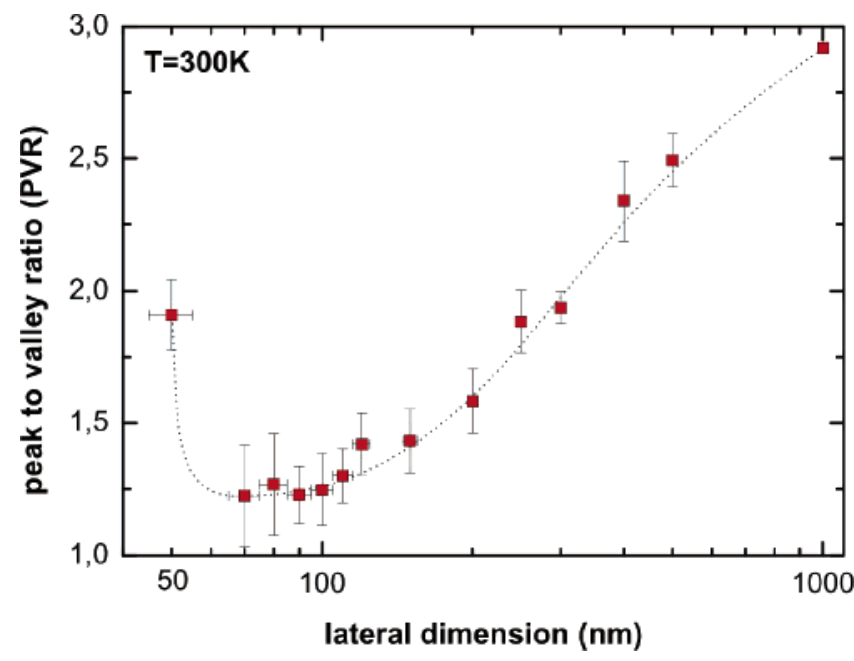

Figure 3. Experimentally determined peak-to-valley ratio (PVR) as a function of the lateral dimension of the nanocolumn. Here, the plotted squares correspond to ensemble averages over multiple devices of the same size. The spread in the column diameters was determined experimentally to be less than $5 \mathrm{~nm}$. (The dotted line is a guide for the eye.)

channel region. Note that a rigorous description of electronic transport in the considered nanostructures would require a 3D quantum kinetic approach. However, for the analysis of the overall potential profile under subthreshold bias condi- tions (i.e., below the transport resonance with still negligible current) the presented semiclassical treatment becomes adequate. Results of the simulation for the 50-nm structure with a bias voltage of $1 \mathrm{~V}$ are shown in Figure 4. Two experimentally observed features can be explained with the help of the simulated potential profile (Figure 4a). First, the shifted peak position in the $I-V$ characteristics for smaller column diameters (Figure 2) can be understood easily by inspection of the potential profile along the vertical center of the nanocolumn (Figure 4b) where we observe the formation of a depletion-induced barrier $(180 \mathrm{meV}$ for the 50-nm structure), which increases with decreasing lateral dimension. Second, by closer inspection, this barrier can be identified as part of a 3D saddle point in the potential profile ${ }^{25}$ with significantly enhanced lateral confinement in the region of the undoped double-barrier structure (see Figure 1). As will be discussed further, the latter explains the improved characteristics for the 50-nm structure directly.

Figure 5a visualizes an idealized scenario (at low temperatures), where the schematic sketch shows the lateral $(x, y)$ depletion regions within the channel as a function of the vertical transport direction $(z)$. The areas at the outer ends on the source (left) and the drain (right) side of the channel are highly doped, resulting in narrow surface depletion regions. Hence, these regions provide a wide channel area, which implies low lateral quantization energies. Furthermore, the undoped region of approximately $18 \mathrm{~nm}$ in the vicinity of the double-barrier layer system (which is located in the center of the channel, see also Figure 1) yields a reduction of the lateral dimension, implying a large lateral quantization energy. Note that the channel at this position of maximum confinement is not completely quenched as one would obtain for a homogeneous undoped system that exhibits overlapping depletion regions. In fact, for a thin undoped region we observe a saddle point in the potential that allows for a solution of the Poisson equation with strong curvature in the (lateral) $x, y$ directions in undoped (space charge free) regions. In Figure 5b, the corresponding electronic energies are plotted as a function of wavenumber $k_{z}$ in the transport direction at three selected positions, $z$. Assuming a 2D harmonic lateral confinement potential $(x, y)$, we obtain equidistantly spaced subband energy spectra with superimposed 1D parabola due to the motion in transport direction (z). Such an approach is based on the assumption of a lateral potential that varies adiabatically along the $z$ direction, implying the conservation of the lateral subband index during electron propagation (preserving the symmetry of the lateral wave function). Furthermore, we assume ballistic transport, which requires energy conservation. The electronic states marked in red on the dispersion curves in Figure $5 \mathrm{~b}$ are the only states that simultaneously fulfill energy and subband index conservation for the considered propagation direction from the left to the right side. All of the other states are reflected by the saddle point of the electrostatic potential. The total energy barrier, $E_{\min }$, is given by the saddle-point height, increased by the difference in the lateral zero point energies. As a crucial condition, we assume that only the lowest lateral mode becomes occupied at the saddle point, 

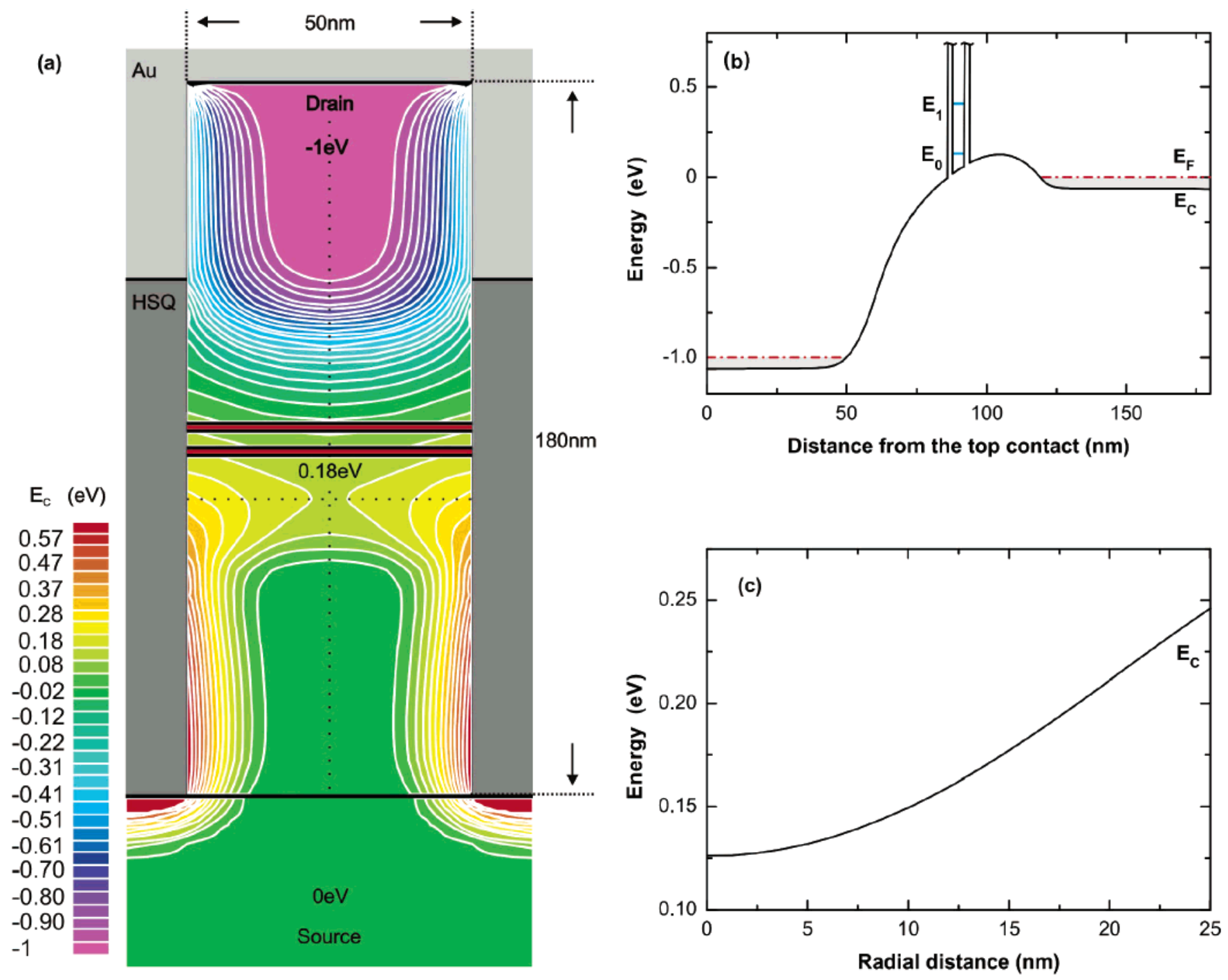

Figure 4. (a) Color plot of the simulated conduction band profile within the 50-nm nanocolumn for subthreshold bias conditions $\left(V_{\mathrm{DS}}=\right.$ $1 \mathrm{~V}$ ). Here, the positive voltage is applied to the top contact with respect to the substrate (bottom contact). Because of the applied voltage, the saddle point (intersection of the dotted lines) is located slightly beneath the double barrier (horizontal red lines). (b) Conduction band profile $E_{\mathrm{c}}(z)$ along the vertical center axis. The local Fermi energies, $E_{\mathrm{F}}$, within the contact regions are represented by red dash-dotted lines, whereas $E_{0}$ and $E_{1}$ denote the lowest two resonances of the RTD. (c) Lateral conduction band profile at the saddle point (along the horizontal dotted line in a), providing an almost harmonic lateral confinement in the center region (with a quantization energy of $\hbar \omega_{0} \approx$ $23 \mathrm{meV}$ ). (Note that the Fermi energy of the source contact is chosen as the energy reference in $\mathrm{b}$ and c.)

that is, $\hbar \omega_{0}>k_{\mathrm{B}} T$ where $\hbar \omega_{0}=\hbar^{2} /\left(m^{*} x_{0}^{2}\right)$ denotes the saddle-point quantization energy with a lateral width of $x_{0}$. Effectively, we end up with a narrow interval $\Delta k$ of transmitted electrons in contrast to the $k_{\mathrm{F}}$ range in the source region. Hence, the single-mode saddle point acts as a $k_{z}$ filter or "collimator" analogous to the quantum point contact in mesoscopic 2D electron gas systems. ${ }^{25,26}$ In this idealized picture, we have assumed conservation of the subband index and hence have neglected lateral mode mixing at the saddlepoint potential. In general, such nonadiabatic effects in combination with the partial occupation of higher modes provide further transport channels through the nanocolumn, weakening the collimation effect to some extent.

As the electrostatic simulations show (see Figure 4a), the position of the saddle point shifts slightly toward the source contact with increasing drain-source bias. Since the doublebarrier resonant tunneling structure is located in the center of the undoped region, the saddle-point energy filter is thus always located in front of the double-barrier layer (as seen from the source contact). In this sense, the saddle point acts as a collimator ( $k_{z}$ prefilter) in front of the resonant tunneling structure, which by itself of course acts as a very selective Fabry-Perot-like filter $\left(k_{z}\right)$. Compared to the situation without the saddle-point formation, where the injected electrons cover a wave vector interval from 0 to $k_{\mathrm{F}}$ (the Fermi wavenumber), we now have a reduced interval $\Delta k$ at $k_{\mathrm{F}}$. Consequently, we expect a significant reduction of the voltage width of the current peak in the $I-V$ characteristics for low temperatures. For room temperature, however, the peak width may be dominated by the thermal broadening on the order of $k_{\mathrm{B}} T$. From the simulated lateral potential within the saddle point (Figure 4c), we obtain a quantization energy of $\hbar \omega_{0} \approx 23 \mathrm{meV}$ for the 50 -nm diode, which is comparable to $k_{\mathrm{B}} T$ at room temperature and, hence, should 


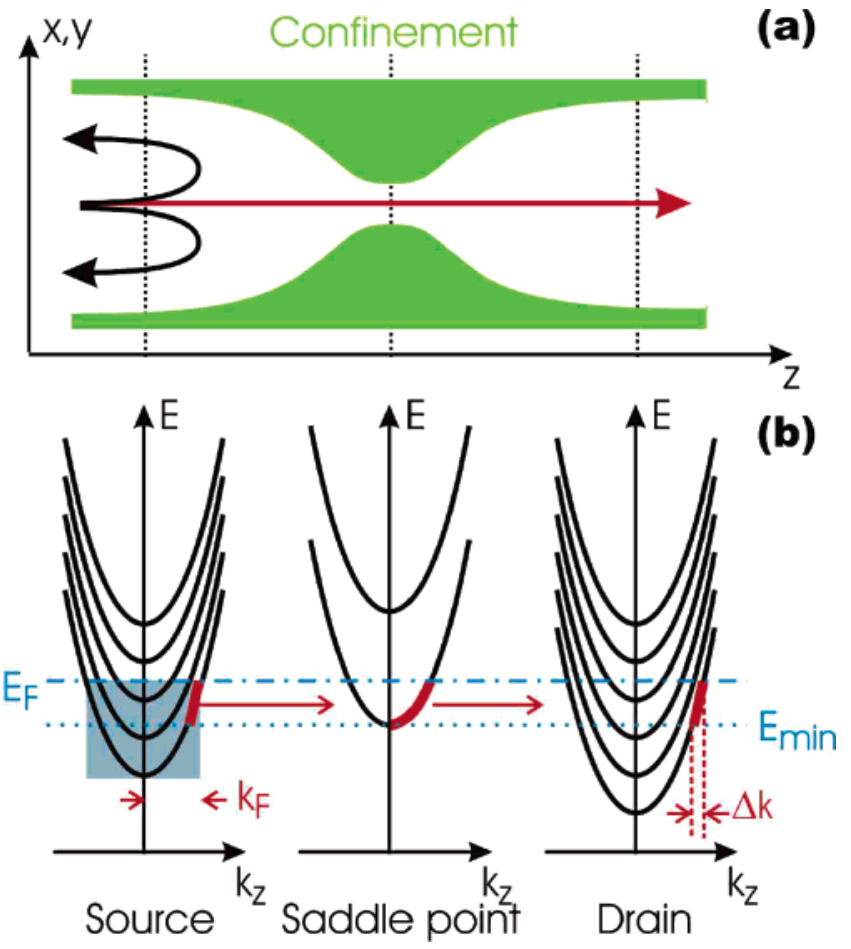

Figure 5. Idealized collimation effect for low temperatures. (a) Schematic sketch of the enhanced lateral confinement (green) due to a saddle-point of the potential as a function of the position, $z$ (i.e., vertical transport direction). The red and black arrows indicate trajectories of transmitted and reflected electrons, respectively. (b) Local electronic subband energies at the source contact, saddlepoint, and drain contact region, respectively, as a function of the wavenumber, $k_{z}$. $E_{\mathrm{F}}$ and $k_{\mathrm{F}}$ denote the source Fermi energy and wavenumber, respectively. $E_{\min }$ is the minimum energy within the saddle point. Transmitted electrons (from source to drain) are visualized by red arrows. Because of the collimation effect for adiabatic transport, only a small interval $\Delta k$ (in contrast to $k_{\mathrm{F}}$ at the source) arrives at the drain region in the shown example.

be relevant for the transport properties. Indeed, the signatures of the discussed collimation behavior can be observed in the experimental results for the nanocolumns with diameters of $50 \mathrm{~nm}$ (see above), where the width for the current peak reads $160 \mathrm{mV}$ compared to $300 \mathrm{mV}$ for the $110-\mathrm{nm}$ diodes (see Figure 2). Furthermore, we expect the peak-to-valley ratio of the device to increase because of a significantly reduced valley current. The latter can be explained in terms of the single-mode (or few-mode) transport situation that results in a reduced phase-space available for scattering events, outweighing the effects of lateral mode mixing (see above) by far. In fact, the experimental results at room temperature for lateral device dimensions of $50 \mathrm{~nm}$ clearly show an improved PVR (see Figure 3). Systems with larger channel diameters (and hence $\hbar \omega_{0} \ll k_{\mathrm{B}} T$ ) in general do not exhibit such features at room temperature due to the lack of a strongly confining saddle point and the missing few-mode injection with collimation in $k_{z}$. In summary, the discussed collimator model explains why our 50-nm diodes show an improved PVR and a sharper resonance compared to the general tendency of down-scaled RTDs with poor PVRs at room temperature (see Figure 3). Future transport investigations in the low temperature regime will certainly reveal more details about the suggested quantization effect. Furthermore, as our fabrication technology is being improved, the scaling behavior in the range of smaller nanocolumn diameters (even below $50 \mathrm{~nm}$ ) will be subject to subsequent studies in this field. These aspects will be addressed in follow-up publications.

In conclusion, we have shown that for nanocolumn devices under conditions where space charge depletion regions are expected to block any reasonable carrier transport, these regions can in fact be used to tailor and improve device performance. For the example of nano-RTDs, collimation of tunneling electrons can be achieved due to the formation of a saddle point in the potential. In this case, the collimation effect leads to unexpectedly sharp resonances in the currentvoltage characteristics in combination with an improved PVR.

Acknowledgment. We thank S. Montanari, M. Goryll, M. Kraemer, and J. Moers for their helpful discussions.

\section{References}

(1) Auth, C. P.; Plummer, J. D. IEEE Electron Dev. Lett. 1997, 18, 74

(2) Hiruma, K.; Yazawa, M.; Katsuyama, T.; Ogawa, K.; Haraguchi, K.; Koguchi, M.; Kakibayashi, H. J. Appl. Phys. 1995, 77, 447.

(3) Cui, Y.; Zhong, Z.; Wang, D.; Wang, W.; Lieber, C. Nano Lett. 2003, 3,149 .

(4) Björk, M. T.; Ohlsson, B. J.; Thelander, C.; Persson, A. I.; Deppert, K.; Wallenberg, L. R.; Samuelson, L. Appl. Phys. Lett. 2002, 81, 4458

(5) Samuelson, L.; Thelander, C.; Björk, M. T.; Borgström, M.; Deppert, K.; Dick, K. A.; Hansen, A. E.; Martensson, T.; Panev, N.; Persson, A. I.; Seifert, W.; Skold, N.; Larsson, L. R.; Wallenberg, L. R. Physica E 2004, 25, 313.

(6) Bakkers, E. P. A. M.; van Dam, J. A.; De Franceschi, S.; Kouwenhoven, L. P.; Kaiser, M.; Verheijen, M.; Wondergem, H.; van der Sluis, P. Nat. Mater. 2004, 3, 769 .

(7) Calarco, R.; Marso, M.; Richter, T.; Aykanat, A. I.; Meijers, R.; van der Hart A.; Stoica, T.; Lüth, H. Nano Lett. 2005, 5, 981.

(8) Reed, M. A.; Randall, R. J.; Aggarwal, R. J.; Matyi, R. J.; Moore, T. M.; Wetsel, A. E. Phys. Rev. Lett. 1988, 60, 535.

(9) Tewordt, M.; Law, V. J.; Kelly, M. J.; Newbury, R.; Pepper, M.; Peacock, D. C.; Frost, J. E. F.; Ritchie, D. A.; Jones, G. A. C. J. Phys.: Condens. Matter 1990, 2, 8969.

(10) Su, B.; Goldman, V. J.; Santos, M.; Shayegan, M. Appl. Phys. Lett. 1991, 58, 747.

(11) Schmidt, T.; Tewordt, M.; Blick, R. H.; Haug, R. J.; Pfannkuche, D.; von Klitzing, K.; Förster, A.; Lüth, H. Phys. Rev. B 1995, 51, 5570

(12) Beton, P. H.; Dellow, M. W.; Main, P. C.; Foster, T. J.; Eaves, L.; Jezierski, A. F.; Henini, M.; Beaumont, S. P.; Wilkinson, C. D. W. Appl. Phys. Lett. 1992, 60, 2508.

(13) Guéret, P.; Blanc, N.; Germann, R.; Rothuizen, H. Phys. Rev. Lett. 1992, 68, 1896.

(14) Goodings, C.; Mizuta, H.; Cleaver, J. R. A.; Ahmed, H. J. Appl. Phys. 1994, 76, 1276.

(15) Austing, D. G.; Honda, T.; Tarucha, S. Semicond. Sci. Technol. 1996 11,388 .

(16) Griebel, M.; Indlekofer, K. M.; Förster, A.; Lüth, H. J. Appl. Phys. 1998, 84, 6718.

(17) Indlekofer, K. M.; Förster, A.; Lüth, H. Physica B 2002, 314, 499.

(18) Chang, L. L.; Esaki, L.; Tsu, R. Appl. Phys. Lett. 1974, 24, 593.

(19) Patkar, M. P.; Chin, T. P.; Woodall, J. M.; Lundstrom, M. S.; Melloch, M. R. Appl. Phys. Lett. 1995, 66, 1412.

(20) Chen, N.-P.; Ueng, H. J.; Janes, D. B.; Woodall, J. M.; Melloch, M. R. J. Appl. Phys. 2000, 88, 309 .

(21) Namatsu, H.; Takahashi, Y.; Yamazaki, K.; Yamaguchi, M.; Nagase, T.; Kurihara, K. J. Vac. Sci. Technol., B 1998, 16, 69.

(22) Trellenkamp, S.; Moers, J.; van der Hart, A.; Kordoš, P.; Lüth, H. Microelectron. Eng. 2003, 67-68, 376. 
(23) Lee, H.-J.; Goo, J.; Kim, S.-H.; Hong, J.-G.; Lee, H.-D.; Kang, H.K.; Lee, S.-I.; Lee, M. Y. Jpn. J. Appl. Phys. 2000, 39, 3924.

(24) Atlas/Blaze, SILVACO International, 2005.

(25) Büttiker, M. Phys. Rev. B 1990, 41, 7906.
(26) Topinka, M. A.; LeRoy, B. J.; Shaw, S. E. J.; Heller, E. J.; Wetervelt, R. M.; Maranowski, K. D.; Gossard, A. C. Science 2000, 289, 2323.

NL051781A 